\title{
Presupuesto por proyectos bajo la perspectiva de sub conjuntos borrosos
}

\section{Fuzzy logic as an advanced tool for budgeting project}

Guillermina Vanessa Sánchez Agila. ${ }^{1}$, Kléber Antonio Luna Altamirano. ${ }^{2}$, Cecilia Ivonne Narváez Zurita. ${ }^{3}$ \& Juan Carlos Erazo Álvarez. ${ }^{4}$

\begin{abstract}
.
DOI: https://doi.org/10.33262/cienciadigital.v3i2.3.484

The present investigation, consists of a model for the elaboration of a budget by projects with the support of the fuzzy logic, for the company of Telecommunications Agila and Sánchez Cía. Ltda., of the city of Loja-Ecuador, the problem lies in the absence of a budgetary model within this entity, complementing itself with the lack of knowledge of the administrators to elaborate a determined budget. The objective of this study is to provide this organization with a new form of calculation based on projects, and with the support of advanced tools that provide fuzzy logic also known as fuzzy logic, such as trapezoidal fuzzy numbers (NBTr), where it is possible to determine correct budget values, trying to limit the uncertainty at each of the established levels, what is explained is detailed within the methodology. The results of this study are intended to establish up to what budget level the new budget model will be able to cover.
\end{abstract}

Keywords: Fuzzy logic, project-based budget, trapezoidal blurry numbers.

\section{Resumen}

La presente investigación, consiste en un modelo para la elaboración de un presupuesto por proyectos con el apoyo de la lógica borrosa, para la empresa de telecomunicaciones Agila y Sánchez Cía. Ltda., de la ciudad de Loja-Ecuador, el problema radica en la ausencia de un modelo presupuestario dentro de esta entidad, complementándose con el desconocimiento de los administradores para elaborar un determinado presupuesto. El

\footnotetext{
${ }^{1}$ Universidad Católica de Cuenca, Maestrante en Contabilidad y Auditoría, Cuenca, Ecuador. gvsancheza880@psg.ucacue.edu.ec

${ }^{2}$ Universidad Católica de Cuenca, Unidad Académica de Administración, Cuenca, Ecuador. klunaa@ucacue.edu.ec

${ }^{3}$ Universidad Católica de Cuenca, Posgrados, Cuenca, Ecuador. inarvaez@ucacue.edu.ec

${ }^{4}$ Universidad Católica de Cuenca, Posgrados, Cuenca, Ecuador. jcerazo@ucacue.edu.ec
} 
objetivo de este estudio es entregar a esta organización una nueva forma de cálculo basado en proyectos, y con el apoyo de herramientas de avanzada que brinda la lógica difusa conocida también como lógica borrosa, tales como los números borrosos trapezoidales (NBTr), en donde se logra determinar, valores correctos presupuestarios, tratando de acotar la incertidumbre en cada uno de los niveles establecidos, lo explicado se detalla dentro de la metodología. Los resultados de este estudio pretenden establecer hasta que nivel presupuestario podrá cubrir el nuevo modelo de presupuesto.

Palabras Clave: Lógica borrosa, presupuesto basado en proyectos, números borrosos trapezoidales.

\section{Introducción}

La empresa de telecomunicaciones Agila y Sánchez Cía. Ltda., es una entidad que brinda soluciones tecnológicas a entornos públicos y privados desde el año 2012, el objeto y finalidad que constan en su escritura pública son la prestación de servicios de telecomunicaciones, electricidad, construcción, diseño y fiscalización de redes de telecomunicaciones. El problema de este estudio, radica en el desconocimiento por parte de los administradores de esta empresa en cómo elaborar un presupuesto, lo cual lo vienen realizando de una manera empírica, únicamente basándose en la experiencia y en un historial de proyectos anteriores, para posteriormente proyectarse de acuerdo a las necesidades de la entidad, el cual arrastra vacíos presupuestarios y estos se infieren al futuro.

El objetivo de este estudio, es contribuir con nuevos conocimientos a los administradores de la entidad, entregando a esta organización una nueva forma de cálculo basado en proyectos, tratando de eliminar estos inconvenientes al agregar como principio la justificación a cualquier gasto que se pretenda dar en el futuro, con el apoyo de la lógica borrosa con aplicación de herramienta de avanzada como los números borrosos trapezoidales (NBTr), se tratará de reducir la incertidumbre y desconfianza en la toma de decisiones a nivel de gestión.

Se determina si los recursos estarán disponibles para ejecutar las actividades y/o se procura la consecución de los mismos se escogen aquellas decisiones que reporten mayores beneficios a la empresa (Burbano, 2005). Se aplican estos estándares en la determinación de presupuesto (materiales, mano de obra y costos indirectos de fabricación). El Presupuesto bajo el enfoque de la lógica borrosa inicia como una idea para la estructura de un modelo presupuestario en la empresa de telecomunicaciones Agila y Sánchez.

En este estudio, se explica cómo elaborar un presupuesto por proyectos bajo la perspectiva de la lógica borrosa, apoyados en herramientas de vanguardia como son los números borrosos trapezoidales (NBTr), los indicados se enuncian en cuádruplos, el número izquierdo enuncia el valor mínimo, el número derecho enuncia el valor máximo logrado, los números centrales representan los valores más posibles que puedan darse dentro del suceso. 
Con respecto a la metodología, se manifiesta de madera precisa la aplicación de instrumentos que brinda la lógica difusa, para recopilar información y elaborar un presupuesto por proyectos. Luego se procede a explicar qué proyectos son de interés dentro de la empresa, y su respectiva evaluación por los expertos conocedores del tema. Finalmente se realiza los cálculos utilizando números borrosos trapezoidales (NBTr) y se presenta los resultados adecuados.

\section{Estado del arte}

El ámbito financiero siempre está en constante actualización es por ello que la sociedad está enfrentando los cambios que se dan continuamente, la empresa de telecomunicaciones Agila y Sánchez Cía. Ltda., se han visto en la necesidad de implementar un modelo de presupuesto mediante el cual se pueda conocer de manera eficiente los ingresos y gastos a incurrir por cada proyecto; para dar a conocer la aplicación de este nuevo modelo de presupuesto se citan los siguientes autores, entre ellos: González, Solís y Alcudia (2010) la ejecución de un proyecto comprende de varias operaciones, por lo mismo la administración se desarrolla a través de la planeación, en esta etapa se pretende prevenir dificultades y anticipar los riesgos, seguido del plan de ejecución, con un eficaz control para que se pueda cumplir con las metas proyectadas.

Cosío (2011) el presupuesto por proyectos tiene la viabilidad y factibilidad positiva en las proyecciones de gastos para cumplir con la ejecución de obras y equipamiento por administración propia o terceros. Moreno (2007) el presupuesto por proyectos no es sólo un instrumento de formulación para definir proyectos, objetivos y metas, su ámbito abarca las actividades de ejecución, seguimiento y evaluación de la gestión empresarial. Toro (2010) en las empresas gubernamentales como en las privadas se observa que la ejecución de un proyecto está encaminada al logro de los objetivos estratégicos de las mismas, el presupuesto por proyectos permite la elaboración de un plan de negocios en el cual se analiza la inversión necesaria para su funcionamiento. Burbano (2005) el presupuesto por proyectos es importante dentro de las empresas que realizan una planificación de sus proyectos a realizar en un tiempo determinado para cubrir con la inversión de todos los procedimientos que requiere e proyecto. Lindegaard y Gálvez (2003) muchos directivos de las empresas sostienen que los presupuestos por proyectos son herramientas fundamentales para ser líderes del mercado, siendo el objetivo de este modelo sustituir el éxito accidental de la gestión de la empresa por criterios profesionales deliberados y bien creados.

Para la gestión institucional, el modelo de un presupuesto por proyectos mediante la lógica borrosa, es una herramienta fundamental para obtener una buena rentabilidad en la empresa. Lotfi Asker Zadeh en 1965 crea conceptos de la lógica difusa y de los subconjuntos borrosos, a través de niveles de pertenencia, proporcionando el origen de la lógica borrosa. Flores y García (2013) afirman: “el modelo de la lógica difusa es diseñado a partir de la correspondencia no lineal entre una o varias variables de entrada y una variable de salida; esto facilita una base desde la cual pueden tomarse decisiones o definir patrones que son representados por valores no exactos" (p. 236). 
También otros autores han aplicado la lógica difusa como un instrumento eficiente para desarrollar la metodología de las investigaciones, entre los cuales tenemos: Trigueros, Sansalvador y Reig (2000) Lógica borrosa y su aplicación a la contabilidad; Arango, Urán, Pérez (2012) La gestión de indicadores empresariales con lógica difusa para la toma de decisiones; Medina (2006) Estado de la cuestión acerca del uso de la lógica difusa en problemas financieros; Díaz, Coba y Navarrete (2017) Lógica difusa y el riesgo financiero. Una propuesta de clasificación de riesgo financiero al sector cooperativo; Gutiérrez (2006) Aplicación de los conjuntos borrosos a las decisiones de inversión; Luna, Tinto, Sarmiento y Cisneros (2018) Implementación de un presupuesto empresarial base cero bajo el enfoque difuso.

Los autores anteriormente enunciados, mediante sus investigaciones han demostrado la eficiencia en la aplicación de la teoría de la lógica difusa, cuyo propósito es de disminuir la perplejidad de la información mejorando la gestión empresarial.

\section{Metodología}

La empresa de telecomunicaciones Agila y Sánchez Cía. Ltda., para cumplir las metas trazadas en la ejecución de sus proyectos, acude a la estructura de un presupuesto apoyado en herramientas de avanzada que ofrece la lógica difusa, para ello es necesario determinar los recursos económicos por cada proyecto, para luego con el apoyo de los números borrosos trapezoidales (NBTr), proyectarse a futuro.

A modo de ejemplo, se estructura las categorías, los proyectos a ejecutarse con sus respectivas asignaciones económicas, para ello se presenta la siguiente tabla.

Tabla 1. Categorías de proyectos

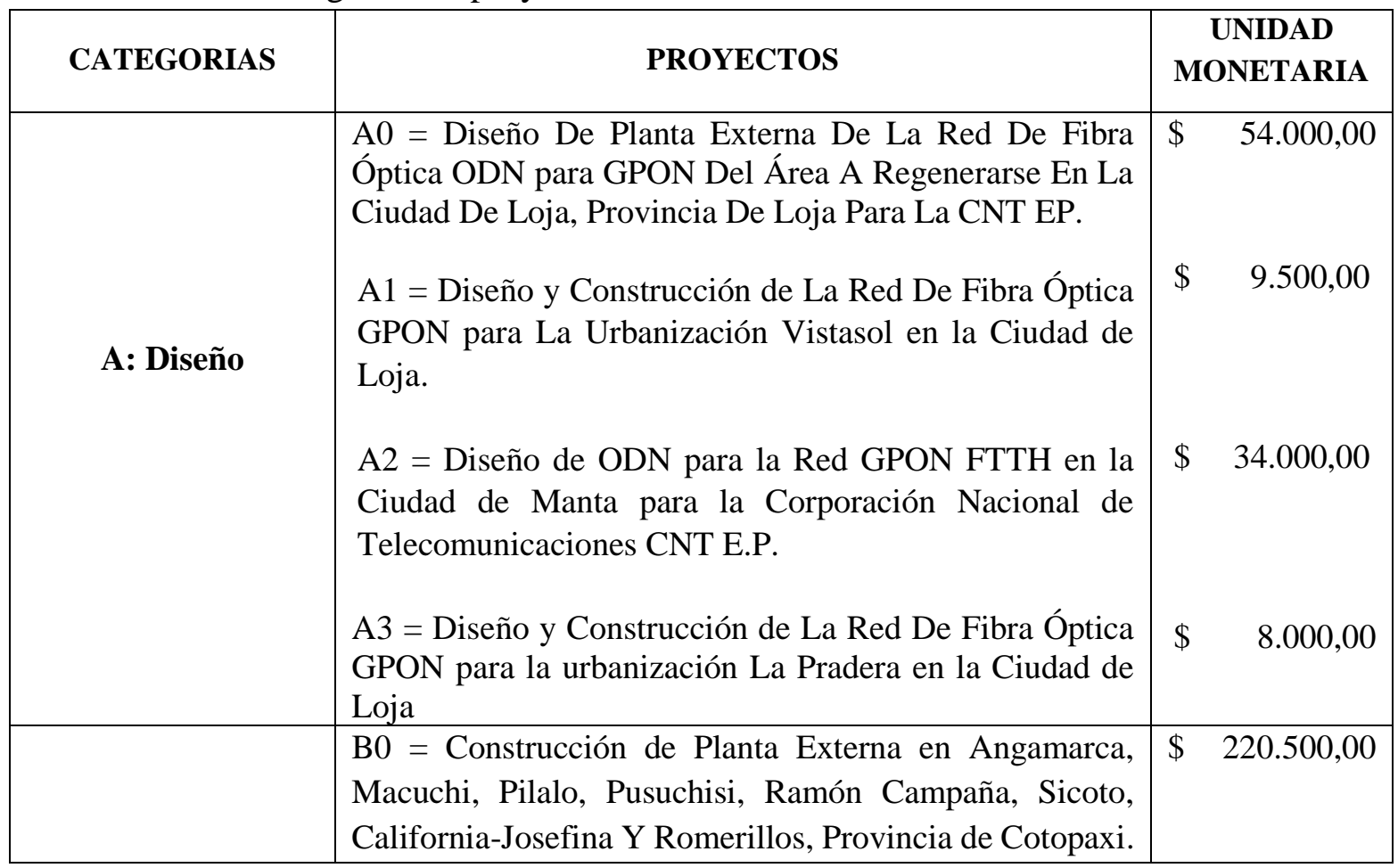




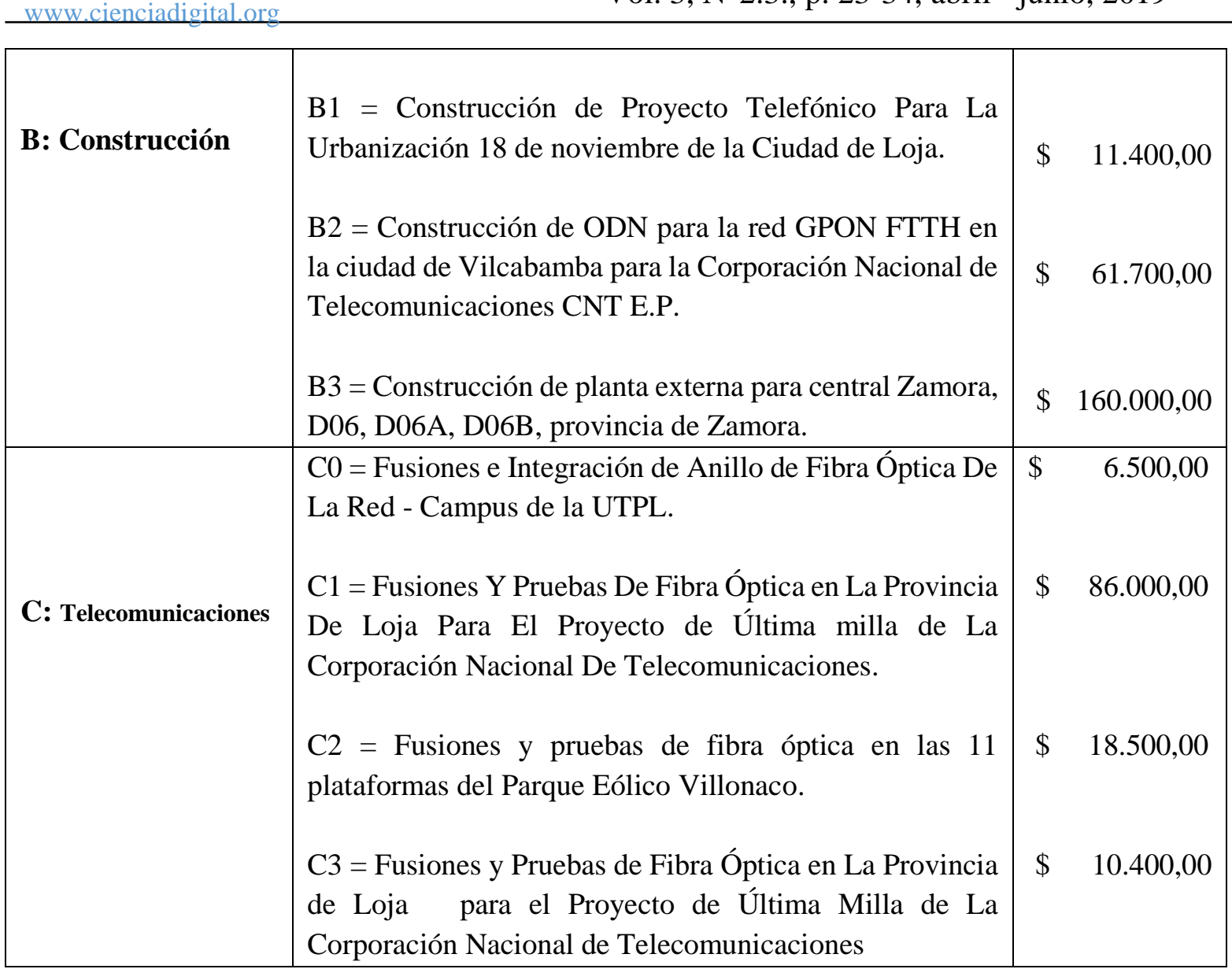

Fuente: Elaboración propia

Como segundo paso, se determina los ingresos generados en un determinado periodo, estos datos se presentan en la siguiente tabla 2.

Tabla 2. Fuentes de ingreso

\begin{tabular}{|l|l|}
\hline $\begin{array}{l}\text { Anticipo del 50\% para ejecución del proyecto } \\
\text { Préstamos a la banca privada }\end{array}$ & $\$ 505.232,65$ \\
& $\$ 380.490,00$ \\
\hline INGRESOS TOTALES ESTIMADOS & $\$ 885.722,65$ \\
\hline
\end{tabular}

Fuente: Elaboración propia

Se realiza la sumatoria del costo de todos los proyectos predispuestos a ejecutarse por la empresa, según tabla 3.

Tabla 3. Cifra Presupuestaria

\begin{tabular}{|l|ll|}
\hline $\mathrm{A}=$ DISEÑO & $\$$ & $105.500,00$ \\
$\mathrm{~B}=$ CONSTRUCCIÓN & $\$$ & $453.600,00$ \\
$\mathrm{C}=$ TELECOMUNICACIONES & $\$$ & $121.400,00$ \\
\hline TOTAL & $\$$ & $680.500,00$ \\
\hline
\end{tabular}

Fuente: Elaboración propia 
Con la información precedente, se recurre a los expertos dentro de la empresa, con la finalidad de determinar valores presupuestarios para el cumplimiento de los proyectos, para ello es necesario recurrir a la escala endecadaria. Kaufmann y Gil-Aluja (1989) afirman: "La introducción de una valuación matizada entre 0 y 1 permite hacer intervenir niveles de verdad en la noción de incidencia. (...) Valores de 0 a 1 (la llamada valuación endecadaria)" (p. 26). La escala endecadaria utilizada para este análisis se presenta en la siguiente tabla.

Tabla 4. Escala endecadaria

\begin{tabular}{|c|c|}
\hline $\begin{array}{c}\text { GRADO DE } \\
\text { PRESUNCIÓN } \\
\boldsymbol{\alpha}\end{array}$ & INCIDENCIA \\
\hline 0 & Bajo \\
\hline 0,1 & Prácticamente bajo \\
\hline 0,2 & Muy bajo \\
\hline 0,3 & Bastante Bajo \\
\hline 0,4 & Más bajo que alto \\
\hline 0,5 & Tan bajo como alto \\
\hline 0,6 & Más alto que bajo \\
\hline 0,7 & Bastante alto \\
\hline 0,8 & Muy alto \\
\hline 0,9 & Prácticamente alto \\
\hline 1 & Alto \\
\hline
\end{tabular}

Fuente: Elaboración propia

Con la información obtenida en base a las respuestas de los expertos funcionarios de la organización, se determina 473.600,00 dólares, en el caso pesimista; y 511.500,00 dólares en el caso optimista, estos valores se expresan en la tabla 5.

Tabla 5. Recursos económicos para el cumplimiento de los proyectos

\begin{tabular}{|l|cc|cr|}
\hline Componentes de los ingresos & \multicolumn{2}{|c|}{$\begin{array}{c}\text { Posición } \\
\text { pesimista }\end{array}$} & \multicolumn{2}{c|}{$\begin{array}{c}\text { Posición } \\
\text { optimista }\end{array}$} \\
\hline $\begin{array}{l}\text { Anticipo del 50\% para } \\
\text { ejecución del proyecto }\end{array}$ & $\$$ & $289.500,00$ & $\$$ & $320.500,00$ \\
Préstamos a la banca privada & $\$$ & $184.100,00$ & $\$$ & $191.000,00$ \\
\hline \multicolumn{1}{|c|}{ TOTALES } & $\$$ & $473.600,00$ & $\$$ & $511.500,00$ \\
\hline
\end{tabular}

Fuente: Elaboración propia

Los números borrosos trapezoidales (NBTr), están constituidos por cuatro números dos extremos y dos centrales, que representan la posición pesimista y optimista, expresados así: ( $\mathrm{a}_{1}$, $\left.\left[\mathrm{a}_{2}, \mathrm{a}_{3}\right], \mathrm{a}_{4}\right)$, la siguiente figura presenta lo explicado 
Figura 1. Número borroso trapezoidal (NBTr)

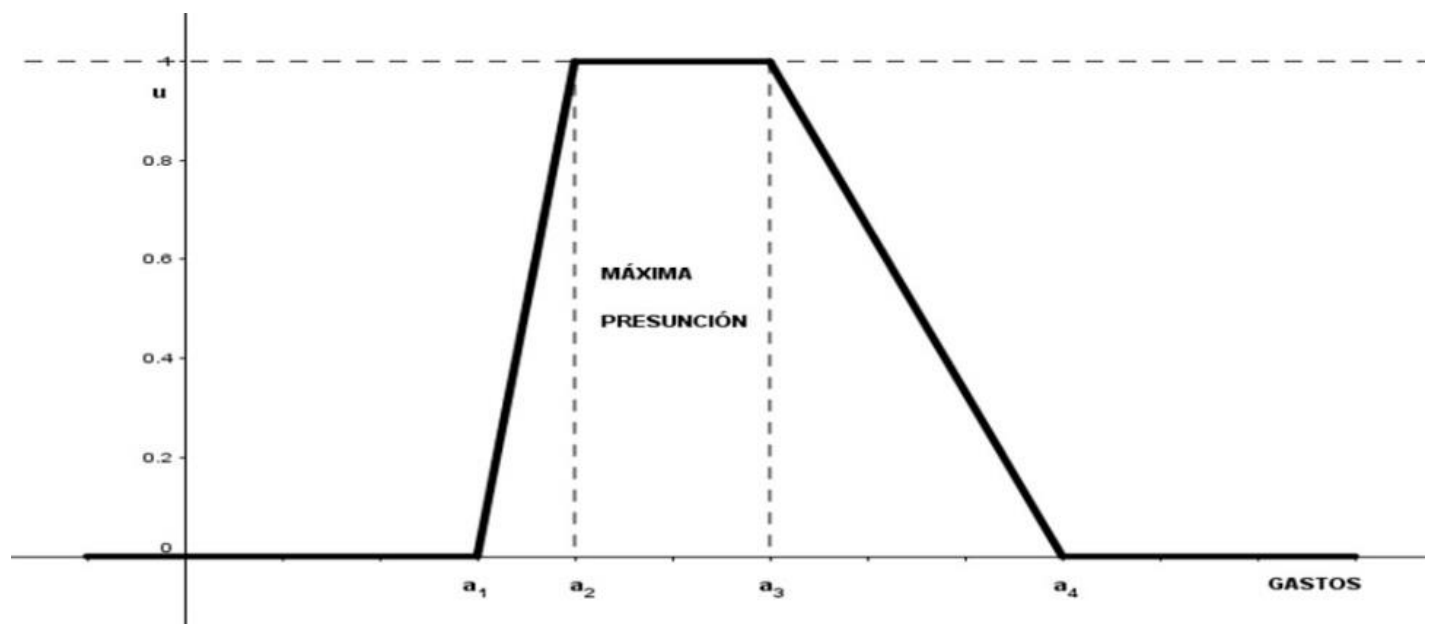

Fuente: Elaboración propia

Los niveles presupuestarios, están representados por cada uno de los proyectos a ejecutarse por la empresa, estos se elaboran de acuerdo a la opinión de los expertos funcionarios de la organización con la finalidad de llegar a cumplir con la ejecución de cada uno de ellos, estos niveles presupuestarios se presentan en la siguiente tabla.

Tabla 6. Niveles Presupuestarios

\begin{tabular}{|c|c|c|c|c|c|}
\hline \multirow{2}{*}{ Niveles } & \multirow{2}{*}{ Proyectos } & \multicolumn{4}{|c|}{ Números borrosos trapezoidales } \\
\hline & & $\mathbf{a}_{1}$ & $\mathbf{a}_{2}$ & $\mathbf{a} 3$ & $\mathbf{a}_{4}$ \\
\hline 1 & X0 & $\$ 47.500$ & $\$ 50.500$ & $\$ 57.500$ & $\$ 60.500$ \\
\hline 2 & $\mathrm{X} 0+\mathrm{X} 1$ & $\$ 57.000$ & $\$ 60.000$ & $\$ 67.000$ & $\$ 70.000$ \\
\hline 3 & $\mathrm{X} 0+\mathrm{X} 2+\mathrm{Z} 0$ & $\$ 63.500$ & $\$ 66.500$ & $\$ 73.500$ & $\$ 76.500$ \\
\hline 4 & $\mathrm{X} 0+\mathrm{X} 2+\mathrm{Z} 0+\mathrm{X} 2$ & $\$ 97.500$ & $\$ 100.500$ & $\$ 107.500$ & $\$ 110.500$ \\
\hline 5 & $\mathrm{X} 0+\mathrm{X} 2+\mathrm{Z} 0+\mathrm{X} 2+\mathrm{Z} 1$ & $\$ 183.500$ & $\$ 186.500$ & $\$ 193.500$ & $\$ 196.500$ \\
\hline 6 & $\mathrm{X} 0+\mathrm{X} 2+\mathrm{Z} 0+\mathrm{X} 2+\mathrm{Z} 1+\mathrm{Y} 0$ & $\$ 404.000$ & $\$ 407.000$ & $\$ 414.000$ & $\$ 417.000$ \\
\hline 7 & $\mathrm{X} 0+\mathrm{X} 2+\mathrm{Z} 0+\mathrm{X} 2+\mathrm{Z} 1+\mathrm{Y} 0+\mathrm{Y} 1$ & $\$ 415.400$ & $\$ 418.400$ & $\$ 425.400$ & $\$ 428.400$ \\
\hline 8 & $\mathrm{X} 0+\mathrm{X} 2+\mathrm{Z} 0+\mathrm{X} 2+\mathrm{Z} 1+\mathrm{Y} 0+\mathrm{Y} 1+\mathrm{Y} 2$ & $\$ 477.100$ & $\$ 480.100$ & $\$ 487.100$ & $\$ 490.100$ \\
\hline 9 & $\mathrm{X} 0+\mathrm{X} 2+\mathrm{Z} 0+\mathrm{X} 2+\mathrm{Z} 1+\mathrm{Y} 0+\mathrm{Y} 1+\mathrm{Y} 2+\mathrm{Z} 2$ & $\$ 495.600$ & $\$ 498.600$ & $\$ 505.600$ & $\$ 508.600$ \\
\hline 10 & $\mathrm{X} 0+\mathrm{X} 2+\mathrm{Z} 0+\mathrm{X} 2+\mathrm{Z} 1+\mathrm{Y} 0+\mathrm{Y} 1+\mathrm{Y} 2+\mathrm{Z} 2$ & $\$ 503.600$ & $\$ 506.600$ & $\$ 513.600$ & $\$ 516.600$ \\
\hline 11 & $\mathrm{X} 0+\mathrm{X} 2+\mathrm{Z} 0+\mathrm{X} 2+\mathrm{Z} 1+\mathrm{Y} 0+\mathrm{Y} 1+\mathrm{Y} 2+\mathrm{Z} 2+\mathrm{Z} 3$ & $\$ 514.000$ & $\$ 517.000$ & $\$ 524.000$ & $\$ 527.000$ \\
\hline 12 & $\mathrm{X} 0+\mathrm{X} 2+\mathrm{Z} 0+\mathrm{X} 2+\mathrm{Z} 1+\mathrm{Y} 0+\mathrm{Y} 1+\mathrm{Y} 2+\mathrm{Z} 2+\mathrm{Z} 3+\mathrm{Y} 3$ & $\$ 674.000$ & $\$ 677.000$ & $\$ 684.000$ & $\$ 687.000$ \\
\hline
\end{tabular}

Fuente: Elaboración propia

Apegados al análisis geométrico, y con el apoyo de los números borrosos trapezoidales (NBTr) que ofrece la lógica difusa, se trazan trapezoides para cada uno de los niveles presupuestarios 
establecidos en la tabla 6, estos representan cada uno de los proyectos planteados con su respectiva restricción presupuestaria, la siguiente figura expresa lo indicado.

Figura 2. Niveles Presupuestados (unidades de mil)

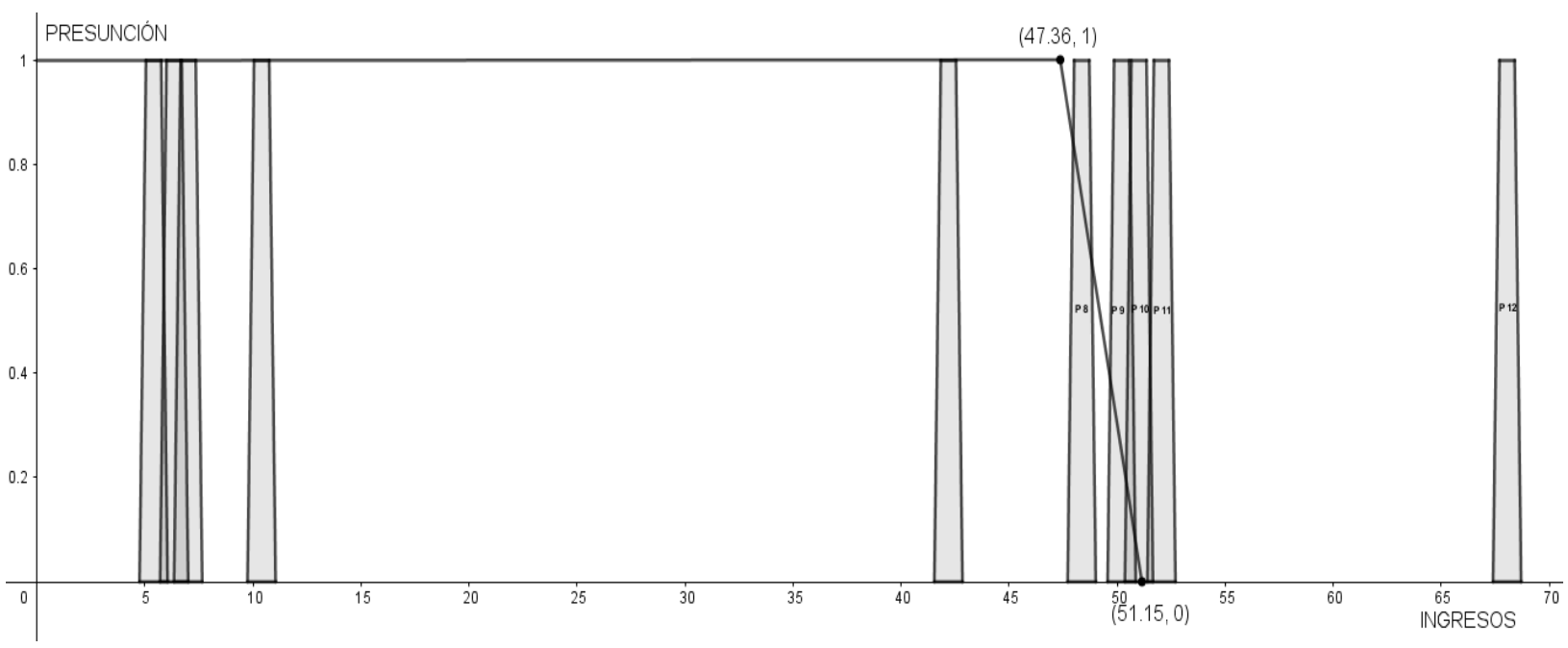

Fuente: Elaboración propia

En la presente figura, se observa que los niveles presupuestados que corresponde a los proyectos desde P1 hasta P7, están dentro del trapezoide, por consiguiente, son aprobados en forma directa, esto quiere decir que la asignación presupuestaria es la adecuada para estos niveles, con relación a los demás niveles presupuestarios, se realizan los cálculos de índices de cobertura para su respectivo análisis.

Figura 3. Análisis del Nivel Presupuestario P8 (unidades de mil)

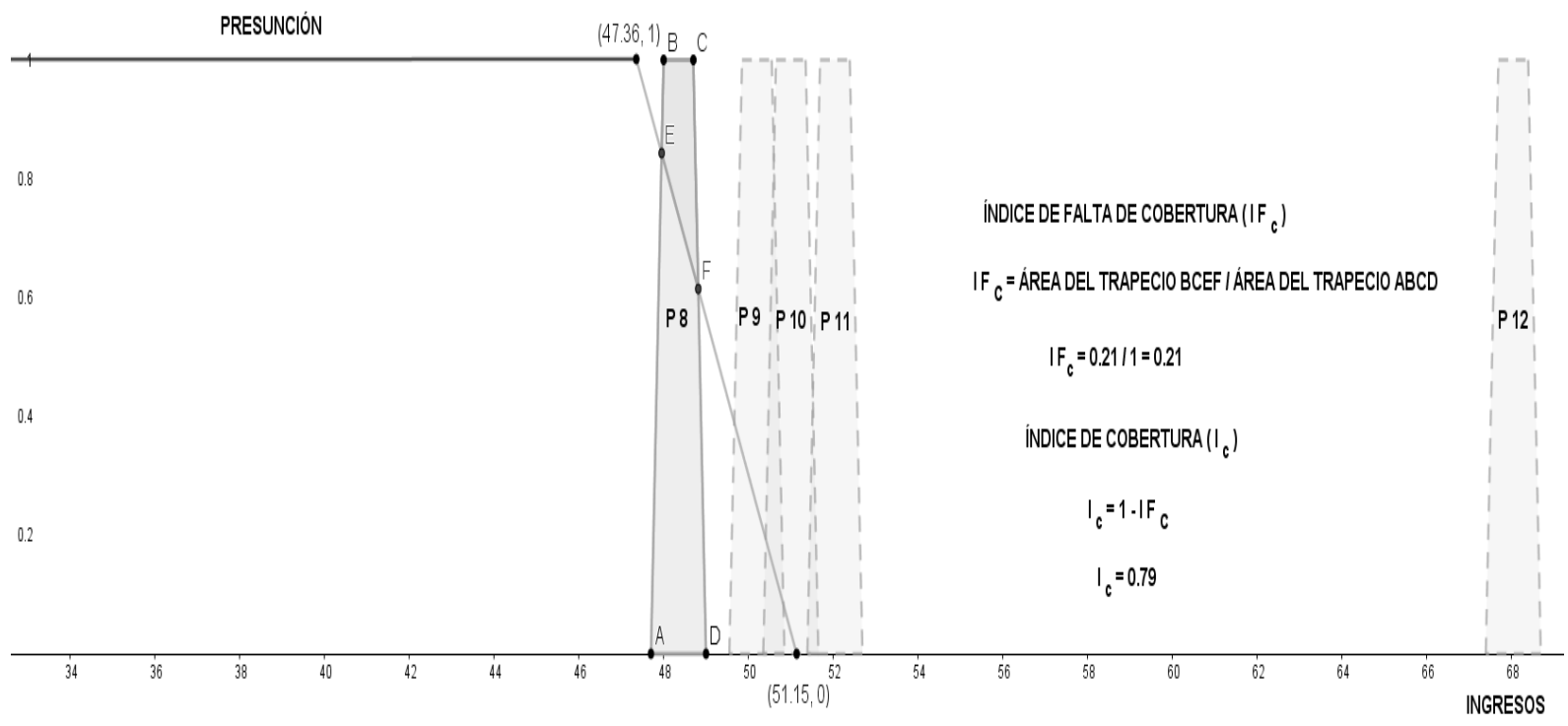

Fuente: Elaboración propia 
El índice de cobertura se refiere al porcentaje que el trapecio cubre al nivel, para determinar este índice, es necesario realizar el cálculo en forma geométrica, para ello se determina el punto de intersección $\mathrm{E}$ y $\mathrm{F}$ con las rectas que pasan por los puntos $\mathrm{AB}$ y $\mathrm{CD}$ respectivamente. Se procede a determinar las áreas de los trapecios AEFD y ABCD, estableciendo el cociente entre ellos, este proceso lleva como nombre IFC (índice de falta de cobertura), su complemento IC = 1 - IFC, desarrollando estos cálculos se obtiene un 79\% de cobertura, su aprobación estaría sujeto al mejor criterio de los directivos de la empresa.

Proceso similar se realiza para los niveles restantes, la figura 4 demuestra estos cálculos.

Figura 4. Análisis del Nivel Presupuestario P9 (unidades de mil)

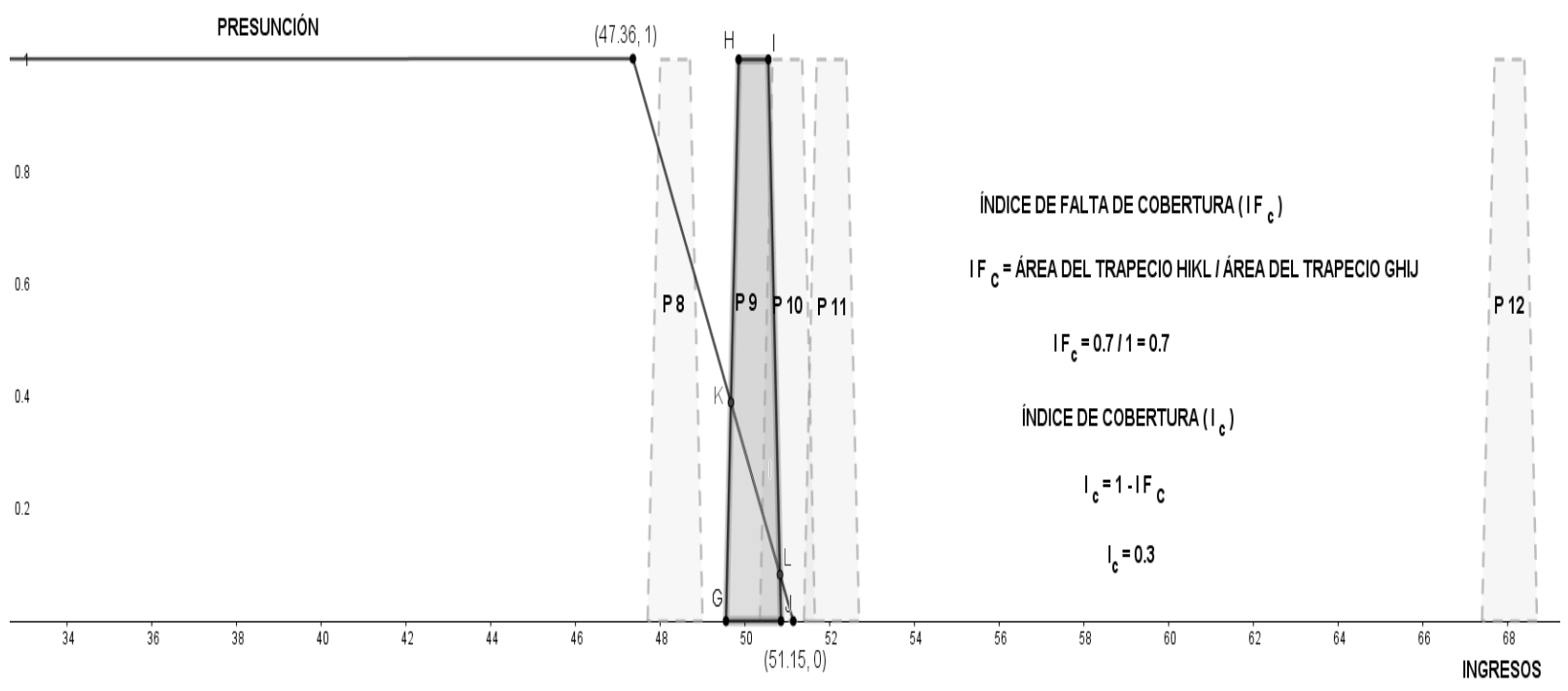

Fuente: Elaboración propia

Siguiendo el mismo proceso anterior, el índice de cobertura para el nivel presupuestario P9 es del 30\%, y el índice de falta de cobertura es del $70 \%$, por tener un nivel bajo de cobertura, este nivel no podría ser aprobado. Los niveles P10, P11 y P12, por estar fuera del índice de cobertura, deberán ser separados del presupuesto, los directivos de la empresa tienen la responsabilidad de analizar y aprobar el respectivo documento presupuestario.

\section{Resultados}

Por encontrarse dentro de la zona trapezoidal los niveles presupuestarios desde P1 a P7, estos podrán ser ejecutados, ya que cuentan con su respectiva asignación económica, el nivel P8 por su nivel de cobertura del $79 \%$ estaría sujeto a una posible aprobación por los directivos de la empresa, en cambio los niveles P10, P11 y P12 por no tener índice de cobertura, estarían sujetos a un rechazo dentro de la ejecución presupuestaria. 


\section{Conclusiones}

Todo presupuesto, está vinculado a la cantidad de recurso económico que se estima necesario para hacer frente a cualquier gasto, desde la perspectiva de elaborar un presupuesto por proyectos, este se refiere a los costos y gastos anticipados que generaría un determinado emplazamiento o proyecto. Realizar un presupuesto basado en proyectos con enfoque en la lógica borrosa utilizando herramientas de vanguardia como son los números borrosos trapezoidales (NBTr), se refiere estimar niveles presupuestarios correctos con el propósito de cumplir con los proyectos establecidos, alcanzado la aceptación de estos niveles dentro del índice de cobertura, aquellos niveles que no están cubiertos por este índice, serán observados por los directivos de la empresa por medio de su análisis para una correcta toma de decisiones.

La presentación de este nuevo diseño de presupuesto a la empresa de Telecomunicaciones Agila y Sánchez Cía. Ltda., de la ciudad de Loja-Ecuador, otorgará a los altos directivos tomar la mejor decisión al momento de aprobar este presupuesto, ya que el mismo permitirá medir el riesgo tratando de reducir la incertidumbre dentro del mismo, con ello se pretende optimizar el recurso económico para una correcta gestión empresarial.

\section{Referencias bibliográficas}

Arango, M., Urán, C. y Pérez, G. (2012). La gestión de indicadores empresariales con lógica difusa para la toma de decisiones. Lámpsakos, 6(8), 47-53. Recuperado de https://dialnet.unirioja.es/servlet/articulo?codigo $=4490568$

Baca, N. y Herrera, H. (2016). Proyectos sociales. Notas sobre su diseño y gestión en territorios rurales. Convergencia, 23 (72), $69-87 . \quad$ Recuperado de http://www.scielo.org.mx/pdf/conver/v23n72/1405-1435-conver-23-72-00069.pdf

Burbano, J. (2005). Presupuestos: enfoque de gestión, planeación y control de recursos. Bogotá. Editorial: McGraw-Hill.

Cosío, J. (2011). Los proyectos y los planes de negocios. PERSPECTIVA, 14 (27), 23 - 45. Recuperado de https://www.redalyc.org/pdf/4259/425941231003.pdf

Díaz, Coba y Navarrete (2017). Lógica difusa y el riesgo financiero. Una propuesta de clasificación de riesgo financiero al sector cooperativo. Contaduría y Administración, 62(5), $\quad 1670 \quad 1686 . \quad-\quad$ Recuperado de http://www.redalyc.org/jatsRepo/395/39557431011/39557431011.pdf

Flores, L. y García, M. (2013). Evaluación de programas públicos mediante lógica difusa: el caso del Programa Hábitat. Política y Cultura, 13(40), 231 - 255. Recuperado de $\mathrm{http}: / / \mathrm{www}$.scielo.org.mx/scielo.php?script=sci_arttext\&pid=S0188-

77422013000200012 
González, J., Solís, R. y Alcudia, C. (2018). Diagnóstico sobre la Planeación y Control de Proyectos en las PYMES de Construcción. Revista de la Construcción, 9 (1), 17 -25. Recuperado de https://scielo.conicyt.cl/scielo.php?script=sci_arttext\&pid=S0718915X2010000100003

Gutiérrez, J. (2006). Aplicación de los conjuntos borrosos a las decisiones de inversión. ADminister, 5(9), 62-85. $\quad$ Recuperado de http://www.redalyc.org/pdf/3223/322327239003.pdf

Lindegaard, E. y Gálvez, G. (2003). Contabilidad de gestión: Presupuestaria y de Costos. Barcelona. Editorial: OCEANO.

Luna, K., Tinto, J., Sarmiento, W. y Cisneros, D. (2018). Implementación de un presupuesto empresarial base cero bajo el enfoque difuso. Revista Ciencia UNEMI, 11(27), 43 -51. Recuperado de http://ojs.unemi.edu.ec/index.php/cienciaunemi/article/view/373

Medina, S. (2006). Estado de la cuestión acerca del uso de la lógica difusa en problemas financieros. Cuadernos de Administración, 19(32), 195 - 223. Recuperado de https://www.redalyc.org/pdf/205/20503209.pdf

Moreno, Z. (2007). Presupuesto por proyectos: un desafío para las universidades venezolanas. Gestión y Gerencia, 1 (1), 73-93. Recuperado de https://dialnet.unirioja.es/servlet/articulo?codigo $=5232226$

Mullor, J., Sansalvador, M., y Trigueros. J. (2000). Lógica borrosa y su aplicación a la contabilidad. Revista española de financiación y contabilidad, 19(103), 83 - 106. Recuperado de https://dialnet.unirioja.es/servlet/articulo?codigo=44342

Perea, M., Sandra, P., Castellanos, S., Heiberg, A., Valderrama, B. y Yosman, J. (2016). Estados financieros previsionales como parte integrante de un conjunto completo de estados financieros en ambiente NIIF. Una propuesta en el marco de la lógica difusa. Actualidad Contable FACES, 19(32),

Toro, F. (2010). Costos ABC y presupuestos: herramientas para la productividad. Bogota. Editorial: Ecoe Ediciones.

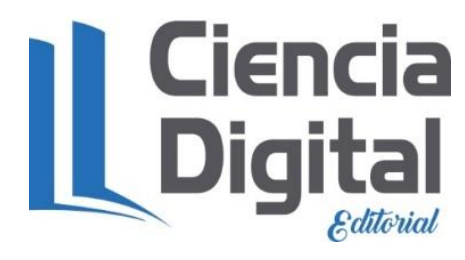




\section{PARA CITAR EL ARTÍCULO INDEXADO.}

Sánchez Agila, G., Luna Altamirano, K., Narváez Zurita, C., \& Erazo Álvarez, J. (2019). Presupuesto por proyectos bajo la perspectiva de sub conjuntos borrosos. Ciencia Digital, 3(2.3), 23-34. https://doi.org/10.33262/cienciadigital.v3i2.3.484

\section{Liencia}

El artículo que se publica es de exclusiva responsabilidad de los autores y no necesariamente reflejan el pensamiento de la Revista Ciencia Digital.

El artículo queda en propiedad de la revista y, por tanto, su publicación parcial y/o total en otro medio tiene que ser autorizado por el director de la Revista Ciencia Digital.
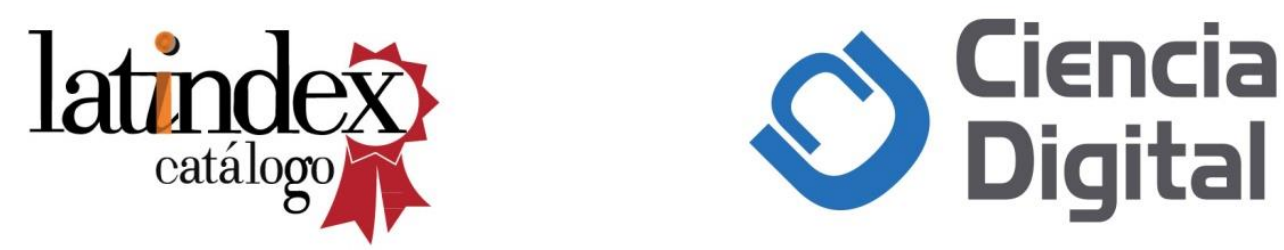